\title{
Computer Simulation of the Skier-Flex Pole Impact in Slalom
}

\author{
Kurt Schindelwig, Peter Kaps, and Werner Nachbauer
}

\begin{abstract}
In skiing the skier-flex pole impact causes a deflection and rotation of the flex pole and a speed loss of the skier. The purpose of the present study was to investigate the effects of skier and pole parameters on time loss, pole deflection, and pole damage speed caused by the skier-pole impact in slalom. Validated finite element models were used for the simulation of the impact. Skier mass, speed and impact height and pole mass, bending stiffness, diameter, and wall thickness were analyzed. Time loss was assessed for seven pole impacts by a simple simulation model of a skier schussing down an inclined plane. From the skier parameters, impact height followed by impact speed showed the highest effect on the skier-pole impulse. The impulse increased with increasing pole mass whereas the effect of bending stiffness was negligible. Time loss could be reduced by lowering the pole mass. However, lowering of pole diameter or wall thickness increased pole deflection enhancing injury risk due to the whiplash effect. Additionally, the reduction of wall thickness decreased pole damage speed with the disadvantage of higher risk of pole fractures. Overall, lowering pole mass for the current impact speeds in World Cup slalom races requires additional investigation. In children and youth races with lower impact speeds than in World Cup races, a pole mass reduction would be possible.
\end{abstract}

Keywords Flex pole $\bullet$ Slalom $\bullet$ Time loss $\bullet$ FE model $\bullet$ Safety aspects $\bullet$ Impact

\section{Introduction}

According to the competition rules of the International Ski Federation World Cup skiers hit about 130 flex poles during a slalom race [1]. Specifications for flex poles were developed by the FIS to guarantee that flex poles behave in the same manner

\footnotetext{
K. Schindelwig $(\bowtie) \bullet$ W. Nachbauer

Department of Sport Science, University of Innsbruck, Innsbruck, Austria

e-mail: kurt.schindelwig@uibk.ac.at

P. Kaps

Department of Engineering Mathematics, University of Innsbruck, Innsbruck, Austria
} 
all over the world, to avoid injury caused by the pole impacts as much as possible, and minimize the risk of pole fracture so that a flex pole is operational over a period of at least 3 years (ca. 8000 passes) [2].

The resistance of the flex pole during impact leads to a speed loss and in consequence to a time loss of the skier. Innerhofer and Nachbauer [3] reported that skiers of low body mass and height have a competitive disadvantage compared to heavier and taller skiers. This disadvantage was more pronounced the more mass and height differed, e.g., speed loss per pole contact was reduced $23 \%$ by increasing body mass from 40 to $90 \mathrm{~kg}$ and reduced $27 \%$ by increasing impact height from 0.6 to $1.2 \mathrm{~m}$.

The impact of the skier causes a deflection of the flex pole that may cause injury to the skier. As a result of the very fast acceleration of the upright pole by the skier impact, the tip of the pole rebounds shortly and may hit the skier in the head-shoulder-back area. This effect-in skiing referred to as the whiplash effect-is stronger for an upright pole with lower bending stiffness and higher mass. There are no injury data available for the whiplash injury mechanism. Bere et al. [4] analyzed 69 injury cases of the alpine skiing World Cup. Gate contact was assumed to contribute directly and/or indirectly to injury in 21 cases. Impacts that contributed indirectly to injury influenced the skier's balance leading to falls. Two injuries were reported for slalom. However, only severe injuries were analyzed.

Pole fracture data was not published. According to informal reports of coaches and pole manufacturers pole impacts by a skier causes fractures in the middle part of the pole and pole impacts to the ground create fractures in the tip region of the pole. Pole fractures during training/competition shorten the training time or extend the competition time. Moreover, flex poles are relatively expensive. In order to avoid fractures in the tip region, some manufactures attach an additional shell inside the upper end of the pole increasing wall thickness. To prevent injuries due to broken poles, FIS requests a ductile fracture behavior to ensure that the pole only snaps without breaking off completely [2].

In the study of Innerhofer and Nachbauer [3], the pole was modeled as a bending beam. The impact was modeled as pure elastic and pole deflection was taken from video images. Accordingly, plastic deformation was not considered. Schindelwig et al. [5] developed a finite element (FE) model of a flex pole. The model was validated using a pendulum impactor. Measured and simulated data correlated well with less than $2 \%$ difference for the impulse and $1.2 \%$ difference for the pole deflection.

Lighter and smaller racers have a considerable disadvantage in competitions due to pole resistance. A reduction of the resistance lessens the disadvantage; however, it may increase injury risk and pole fractures. Thus, the purpose of the present study was to investigate the effects of skier and flex pole parameters on time loss, pole deflection, and pole damage speed caused by the skier-pole impact in slalom. 


\section{Methods}

\subsection{Finite Element Modeling and Simulation}

The applied finite element (FE) model of the flex pole was described in Schindelwig et al. [5]. The upright pole was discretized with S4R general quadratic membrane shell elements. Isotropic elastic and plastic material behaviors were used. The bending device was modeled by bushing connector elements with linear elastic behavior. A horizontally moving impactor was used to reproduce the impact by the skier. The impactor was modeled as a cylinder with a diameter of $0.1 \mathrm{~m}$ and a length of $0.2 \mathrm{~m}$. Contact with the flex pole occurs mostly with the ski pole or with the guard of the ski pole (Fig. 1). Since the ski pole is often made of aluminum, an aluminum cylinder was used for the impactor. Isotropic elastic material behavior with a typical Young's modulus for aluminum of $69 \mathrm{GPa}$ [6] was used for the solid extrude elements. The impact mass was set to the skier's mass. Videos recorded with a high-speed camera (frame rate $200 \mathrm{fps}$, Sony NEX-FS700K, Sony, Tokyo, Japan) of two women and two men World Cup slalom races in the 2014/15 season showed that the impact height was in average $44 \%$ of the skier's height. Accordingly, impact height of the first contact between impactor and flex pole was set to $44 \%$ of the skier's height.

The FE simulations were performed in an explicit dynamic analysis using Abaqus explicit 6.12-1 (SIMULIA, Dassault Systèmes, Providence, Rhode Island, US). For element types, the settings "standard" from the element library and "reduced integration" were selected. The normal behavior was used for the contact properties with the "Hard" contact for the pressure-overclosure. Based on the analysis of the above-mentioned high-speed videos ground contact of the pole occurred about $0.1 \mathrm{~s}$ after initial pole contact. Thus, simulations were stopped after $0.1 \mathrm{~s}$.
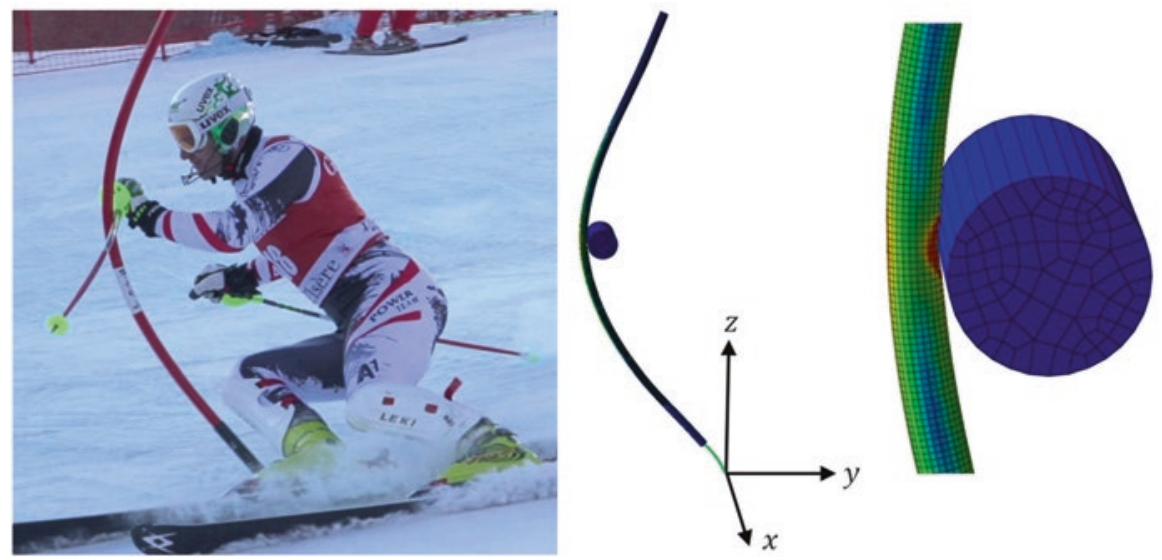

Fig. 1 (Left) picture of the skier-flex pole impact, schematic illustrations of the FE model's flex pole and impactor: (center) overall representation with coordinate system and (right) flex pole and the impactor in detail 


\subsection{Impulse and Speed Loss}

The impulse $p$ was calculated by the speed loss $\Delta v$ of the impact cylinder and its mass $m$ with $p=m \bullet \Delta v$. The speed loss $\Delta v$ of the impact cylinder was determined by the FE simulations for varied skier and pole parameters. Impact height was varied in increments of $0.1 \mathrm{~m}$ from 0.3 to $1.6 \mathrm{~m}$, impact mass in increments of $10 \mathrm{~kg}$ from 30 to $90 \mathrm{~kg}$, and impact speed in increments of $1 \mathrm{~m} / \mathrm{s}$ from 5 to $21 \mathrm{~m} / \mathrm{s}$. Pole mass was varied in increments of $100 \mathrm{~g}$ from 200 to $800 \mathrm{~g}$ by changing pole density. Bending stiffness was varied in increments of $10 \mathrm{Nm}^{2}$ from 20 to $80 \mathrm{Nm}^{2}$ by changing Young's modulus. Only one parameter was varied while the others were kept constant as follows: pole diameter $31 \mathrm{~mm}$ (d31), wall thickness $3 \mathrm{~mm}$ (w3), body mass $90 \mathrm{~kg}$, impact height $0.84 \mathrm{~m}$ (skier "Tall”, Table 1), impact speed $13 \mathrm{~m} / \mathrm{s}$, pole density $1160 \mathrm{~kg} / \mathrm{m}^{3}$, and pole length $1.74 \mathrm{~m}$.

Additionally, all combinations of pole diameters of 25, 27, 29, and $31 \mathrm{~mm}$ and wall thicknesses of 2, 2.5, and $3 \mathrm{~mm}$ were analyzed. The chosen pole diameters were within the limits of $25-32 \mathrm{~mm}$ requested by the FIS [2]. Pole mass was calculated from diameter $d$, wall thickness $w$, length $l$, and densitiy $\rho$ of the pole with

$$
m_{u p}=\left(d \cdot w-w^{2}\right) \cdot \pi \cdot l \cdot \rho .
$$

Young's modulus was set to $2.45 \mathrm{GPa}$ for all poles [5]. Bending stiffness $B_{\text {up }}$ was calculated from diameter $d$, wall thickness $w$, and Young's modulus $E$ with

$$
B_{u p}=E \cdot\left(\frac{d^{4}-(d-2 \cdot w)^{4}}{64}\right)
$$

\subsection{Time Loss}

Speed loss $\Delta v$ of the impact cylinder was obtained by the FE simulation. From speed loss per impact, time loss for seven pole impacts during a straight run on inclined planes of $5^{\circ}$ and $20^{\circ}$ was calculated. For this, the equation of motion of a mass point with initial speed of $13 \mathrm{~m} / \mathrm{s}$, gliding distance of $42 \mathrm{~m}$, and coefficient of snow friction 0.02 was solved. The drag for the skier "Tall" was set to $0.6 \mathrm{~m}^{2}$ according to wind tunnel measurements [7]. Drag was decreased proportional to the mass of the skiers "Small" and "Medium" (Table 1) to 0.2 and $0.4 \mathrm{~m}^{2}$ so that the acceleration of the three skier types was identical and time loss was only caused by the skier-pole impacts. The speed of a skier after impact was set to the speed before impact minus speed loss.

Table 1 Body mass, body height, and impact height of the skiers "Small,"

"Medium," and "Tall"

\begin{tabular}{l|c|c|r}
\hline Skier & Small & Medium & Tall \\
\hline Body mass $(\mathrm{kg})$ & 30 & 60 & 90 \\
\hline Body height $(\mathrm{cm})$ & 130 & 160 & 190 \\
\hline Impact height $(\mathrm{cm})$ & 58 & 71 & 84 \\
\hline
\end{tabular}


Fig. 2 Schematic illustration of the maximum deflection

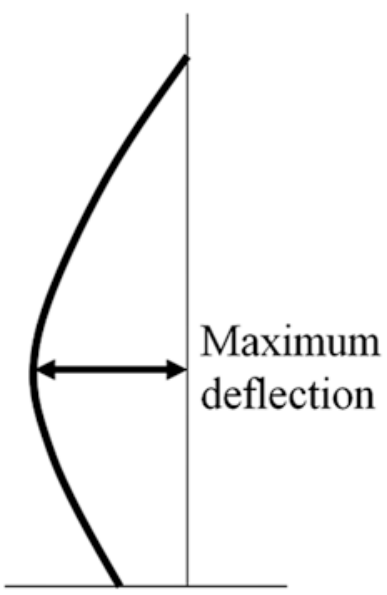

\subsection{Pole Deflection}

The FE simulation provided the 3D coordinates of 35 nodes along the pole in increments of $5 \mathrm{~cm}$ from initial pole contact to ground contact. From the 3D coordinates, the maximum horizontal distance between pole tip and nodes of the pole of the first deflection phase in the running direction (Fig. 2) was determined. This distance was denoted as maximum deflection, which is a measure of the whiplash effect.

\subsection{Pole Damage Speed}

Pole damage speed was defined as the lowest impact speed causing a reduction of the pole diameter of more than $3 \mathrm{~mm}$ at $0.1 \mathrm{~s}$ after initial pole impact. Plastic deformation accounted for this diameter reduction. For the determination of the damage speed, the impact speed was increased in increments of $1 \mathrm{~m} / \mathrm{s}$ starting with $8 \mathrm{~m} / \mathrm{s}$ until an apparent damage occurred. To simulate an unfavorable load case impact height was lowered to $0.5 \mathrm{~m}$ (shin guard impact) and impact mass of $90 \mathrm{~kg}$ was chosen.

\section{Results}

\subsection{Effect of Skier and Pole Parameters on Impulse}

The largest effect on the impulse was observed for impact height followed by impact speed. By lowering impact height from 1.6 to $0.3 \mathrm{~m}$ the impulse increased from 4 to 35 Ns. The effect of body mass on the impulse was negligible. An increase of impact 

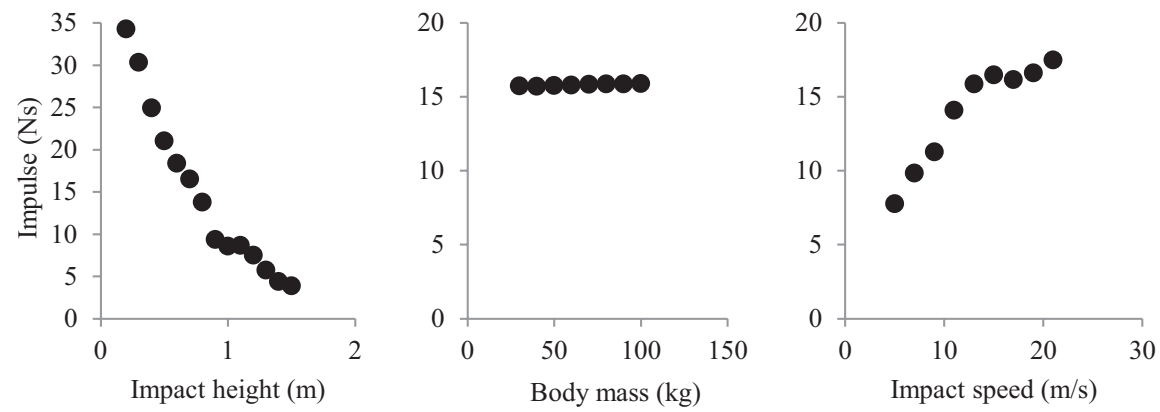

Fig. 3 Impulse for different (left) impact heights, (center) body mass, and (right) impact speeds (flex pole diameter $31 \mathrm{~mm}$ and wall thickness $3 \mathrm{~mm}(\mathrm{~d} 31-\mathrm{w} 3)$ )
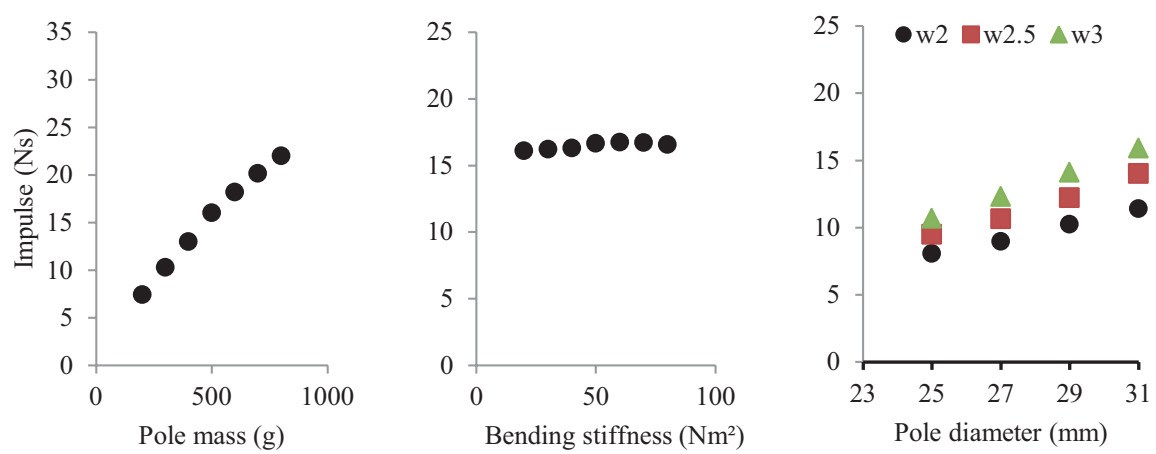

Fig. 4 Impulse for different (left) pole mass and (center) bending stiffness (skier "Tall"), and (right) pole diameter and wall thickness (w) (skier "Tall")

speed from 5 to $21 \mathrm{~m} / \mathrm{s}$ resulted in an increase of the impulse from 8 to 18 Ns. This increase was nearly linear from 5 to $15 \mathrm{~m} / \mathrm{s}$; above $15 \mathrm{~m} / \mathrm{s}$ almost no increase was observed (Fig. 3).

In Fig. 4, the effect of pole mass, bending stiffness, pole diameter, and wall thickness on the impulse are presented for the impact with skier "Tall." The increase of pole mass from 200 to $800 \mathrm{~g}$ caused an increase of the impulse from 8 to $22 \mathrm{Ns}$. Only minor changes of the impulse were observed for bending stiffness between 20 and $80 \mathrm{Nm}^{2}$. The impulse increased approximately linearly with pole diameter as well as with wall thickness (Fig. 4).

The variation of pole diameter and wall thickness varied pole mass and bending stiffness as listed in Table 2. Pole mass and bending stiffness ranged from 290 to $530 \mathrm{~g}$ and from 23 to $64 \mathrm{Nm}^{2}$. 
Table 2 Pole mass and bending stiffness for different pole diameters and wall thicknesses. Material density was $1160 \mathrm{~kg} / \mathrm{m}^{3}$ and Young's modulus $2.45 \mathrm{GPa}$

\begin{tabular}{|c|c|c|c|c|c|c|c|c|c|c|c|c|}
\hline Diameter (mm) & 25 & 27 & 29 & 31 & 25 & 27 & 29 & 31 & 25 & 27 & 29 & 31 \\
\hline $\begin{array}{l}\text { Wall thickness } \\
(\mathrm{mm})\end{array}$ & \multicolumn{4}{|l|}{2} & \multicolumn{4}{|l|}{2.5} & \multicolumn{4}{|l|}{3} \\
\hline Pole mass (g) & 290 & 320 & 340 & 370 & 360 & 390 & 420 & 450 & 420 & 460 & 490 & 530 \\
\hline $\begin{array}{l}\text { Bending } \\
\text { stiffness }\left(\mathrm{Nm}^{2}\right)\end{array}$ & 23.6 & 30.3 & 38.1 & 47.2 & 27.7 & 35.7 & 45.2 & 56.1 & 31.3 & 40.5 & 51.4 & 64.1 \\
\hline
\end{tabular}

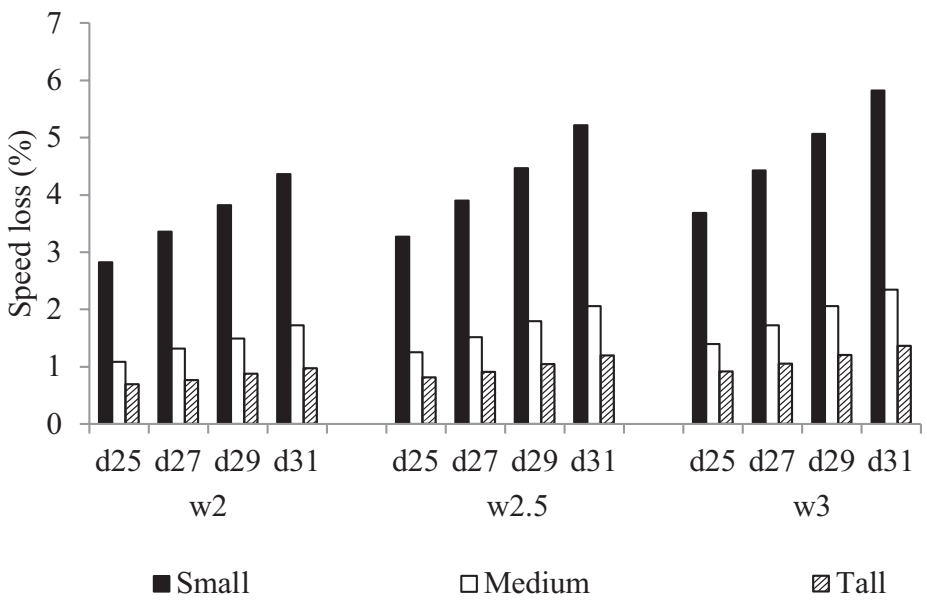

Fig. 5 Speed loss for skiers "Small," "Medium," and "Tall" by impact with different pole diameters and wall thicknesses

\subsection{Effect of Pole Diameter and Wall Thickness on Speed and Time Loss}

The highest speed loss of 5.8\% was calculated for the impact of skier "Small" with the pole diameter $31 \mathrm{~mm}$ and wall thickness $3 \mathrm{~mm}$ (d31-w3). Reducing both the pole diameter from 31 to $25 \mathrm{~mm}$ and the wall thickness from 3 to $2 \mathrm{~mm}$ reduced speed loss to $2.8 \%$. Speed loss for skier "Tall" with poles d31-w3 and d25-w2 was 1.4 and $0.7 \%$, respectively (Fig. 5).

The running time without pole impacts for straight schussing on the $42 \mathrm{~m}$ slope with $5^{\circ}$ inclination was $3.20 \mathrm{~s}$. Seven impacts with d31-w3 resulted in time losses of 0.87, 0.29, and 0.16 s for the skiers "Small," "Medium," and "Tall,, respectively. These time losses were nearly halved for pole $\mathrm{d} 25-\mathrm{w} 2$. On the steeper slope of $20^{\circ}$, time losses were reduced to about $50 \%$ due to higher acceleration of the skiers. In Fig. 6, the time loss differences between the "Small" and "Medium" skiers and "Medium" and "Tall" skiers are presented for seven impacts on the flatter slope of $5^{\circ}$. 


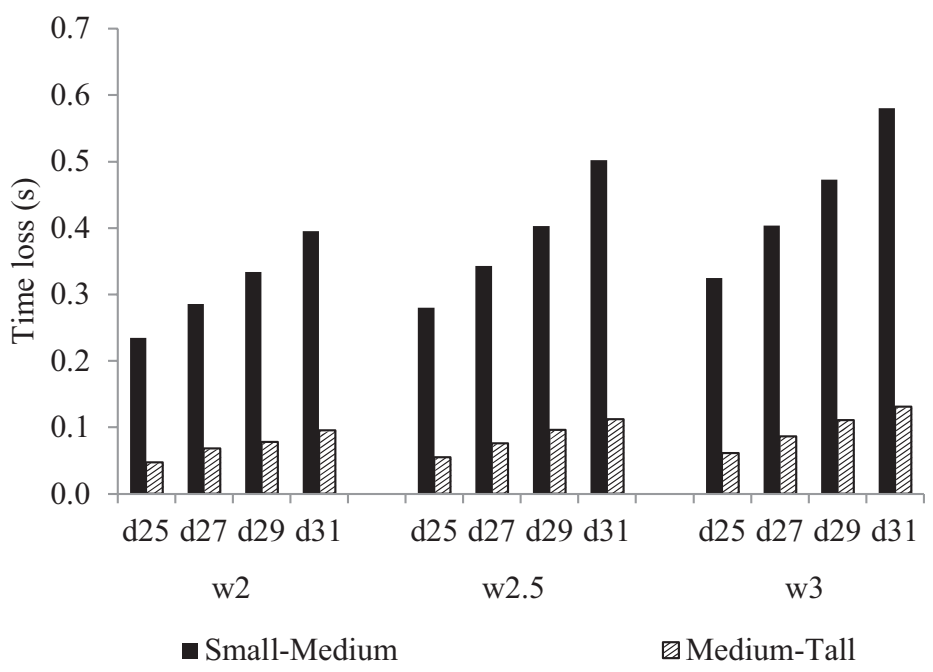

Fig. 6 Time loss differences of skiers "Small"-"Medium" and "Medium"-"Tall" caused by seven impacts during a straight run on a $42 \mathrm{~m}$ long slope with $5^{\circ}$ slope inclination. Flex pole diameter and wall thickness were varied

Reducing both the pole diameter from 31 to $25 \mathrm{~mm}$ and the wall thickness from 3 to $2 \mathrm{~mm}$ reduced the differences of the time losses between skier "Small" and "Medium" from 0.58 to 0.24 s and between "Medium" and "Tall" from 0.13 to $0.05 \mathrm{~s}$.

\subsection{Effect of Pole Diameter and Wall Thickness on Pole Deflection and Pole Damage Speed}

An increase of the pole diameter from 25 to $31 \mathrm{~mm}$ reduced the maximum deflection by about $0.1 \mathrm{~m}$ for all wall thicknesses for skier "Small" and "Medium". Therewas no effect observed of the pole diameter on the maximum deflection for skier "Tall" (Fig. 7 left). The effect of wall thickness on maximum deflection was small. The highest maximum deflection occurred for skier "Small" and the lowest for skier "Tall." The correlation between maximum deflection and impact speed was approximately linear with a gradient of $0.023 \mathrm{~s}$ (Fig. 7 right).

Pole damage speed increased clearly with decreasing pole diameter and increasing wall thickness. For the same wall thickness, a decrease of the pole diameter from 31 to $25 \mathrm{~mm}$ increased the damage speed from 10 to $16 \mathrm{~m} / \mathrm{s}$ (w2), from 15 to $19 \mathrm{~m} / \mathrm{s}$ (w2.5), and from 17 to $20 \mathrm{~m} / \mathrm{s}$ (w3) for wall thickness of 2, 2.5 and $3 \mathrm{~mm}$, 


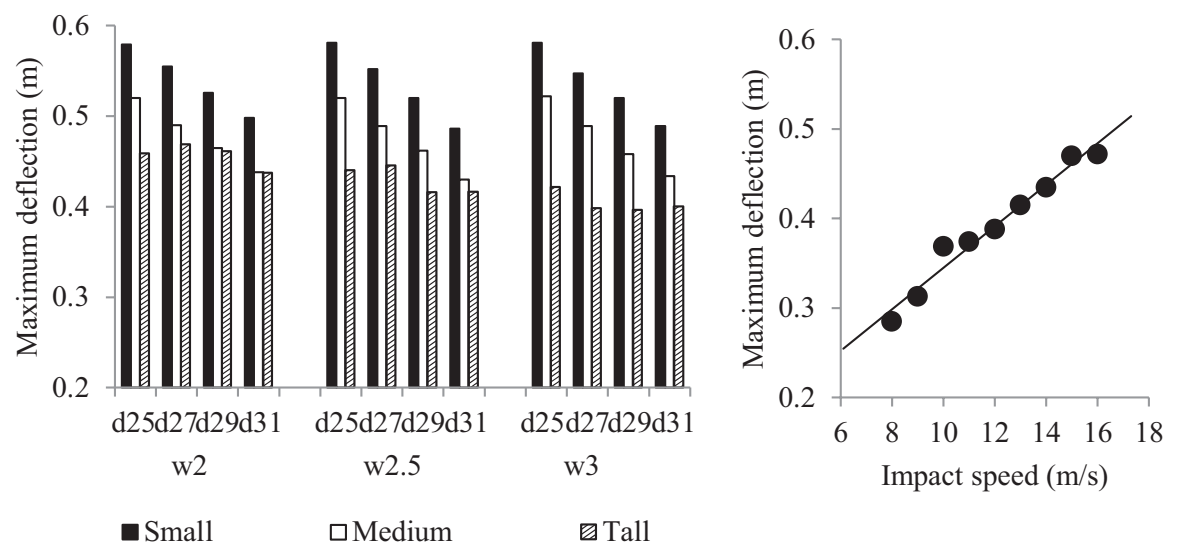

Fig. 7 Maximum deflection for skiers "Small," "Medium," and "Tall" (left) by impact with different pole diameters and wall thicknesses (impact speed $13 \mathrm{~m} / \mathrm{s}$ ), and (right) for impact speeds $8-16 \mathrm{~m} / \mathrm{s}$ (skier "Tall" with pole d31-w3)

Table 3 Damage speed for different pole diameters and wall thicknesses (body mass $90 \mathrm{~kg}$, impact height $0.5 \mathrm{~m}$ )

\begin{tabular}{|l|l|l|l|l|l|l|l|l|l|l|l|l|l}
\hline Diameter $(\mathrm{mm})$ & 25 & 27 & 29 & 31 & 25 & 27 & 29 & 31 & 25 & 27 & 29 & 31 \\
\hline Wall thickness (mm) & 2 & \multicolumn{10}{|l|}{} & 2.5 \\
\hline Damage speed (m/s) & 16 & 14 & 13 & 10 & 19 & 17 & 16 & 15 & 20 & 21 & 19 & 17 \\
\hline
\end{tabular}

respectively. An increase of the wall thickness by $0.5 \mathrm{~mm}$ increased the damage speed between 2 and $3 \mathrm{~m} / \mathrm{s}$ for the different pole diameters (Table 3 ).

\section{Discussion}

The effect of skier and pole parameters on the impulse between skier and pole was determined. For this purpose, skier parameters were varied in a range representatively for athletes of age 10 to adults. Pole parameters were varied reasonably adjusted beyond the limits permitted by the FIS specifications. The impact height of the skier showed the largest effect on impulse. The impulse was about nine times higher at $0.3 \mathrm{~m}$ than at $1.6 \mathrm{~m}$. In addition, impact speed affected the impulse considerably. There was a nearly directly proportional relationship up to an impact speed of $15 \mathrm{~m} / \mathrm{s}$; above $15 \mathrm{~m} / \mathrm{s}$, impulse was independent of speed. Commonly, the skierpole impact consisted of three successive contacts which were also detected in the high-speed videos from World Cup races. In the simulations, the durations of the three contacts were about 10, 5, and $14 \mathrm{~ms}$ for the flex pole d31-w3 and the second 
and third contact was about 39 and $55 \mathrm{~ms}$ after initial contact, respectively. At speeds up to $15 \mathrm{~m} / \mathrm{s}$ almost two thirds of the impulse was generated by the second and third contact. At higher speeds, the first contact resulted in an increased inclination of the pole, reducing the impact during the second and third contact and causing the described speed independency. Unexpectedly, the impulse was nearly independent of the skier's mass. The reason for the independence is the low ratio of the pole mass $(500 \mathrm{~g})$ to the skier's mass. The ratio was lower than $1 / 60$ for the analyzed skiers. The independence of the impulse from the skier's mass leads to an indirectly proportional relationship between mass and speed change of the skier. A reduction of the mass by half doubles the speed loss of a skier.

The impulse was strongly affected by pole mass in a directly proportional manner while the effect of bending stiffness was negligible. For the variation of pole mass and bending stiffness unrealistic values of pole density and Young's modulus were assumed. Mass and bending stiffness of poles are strongly influenced by pole geometry, which is given by diameter, wall thickness, and length. Since geometry affects both mass and bending stiffness, it is in reality not possible to vary one without altering the other. However, the impulse changes presented for different pole diameters and wall thicknesses by keeping the material properties constant were mainly caused by changes of the pole mass. For instance, pole d25-w2 had half the mass of pole $\mathrm{d} 31$ w3, consequently, also the impulse of the pole d25-w2 was half of the pole d31-w3.

Speed loss was calculated through simulations of impacts between different poles and skiers classified as "Small," "Medium," and "Tall." To assess the practical relevance of the speed loss in a slalom competition, the time loss of four $42 \mathrm{~m}$ long flat course sections with seven pole contacts each (two sections per slalom run) was calculated. These calculations revealed that, as compared to skier "Medium," skier "Small" would lose 0.86 and $2.32 \mathrm{~s}$ for poles d25-w2 and d31-w3, respectively. In children's races, body weight and height may differ by a magnitude similar to that between the skiers "Small" and "Medium" considered in our simulations. Assuming a $5.6 \mathrm{~s}$ time difference between rank 1 and 10 (average of six children mastership races) [8], the skier "Small" would lose four positions in the ranking using pole d31-w3 but only one position using pole d25-w2. In World Cup races, usage of pole d31-w3 (World Cup standard) results in a time difference of $0.56 \mathrm{~s}$ between skiers "Medium" and "Tall." This is equivalent to a competitive disadvantage of two positions.

The results revealed that from a view of fairness the reduction of the pole mass would be beneficial. Since the density of polycarbonate lies in a narrow range, mass reduction can only be accomplished by lowering pole length, pole diameter, and/or wall thickness. The reduction of pole length from 1.8 to $1.6 \mathrm{~m}$ was realized in Austria for children competitions younger than 12 years [9]. For children of age 12 and older a length reduction was in discussion, however, it was discarded due to the risk of fascial injury through contact with the tip of the pole. Lowering of pole diameter or wall thickness increases maximum pole deflection, which enhances injury risk since the upper pole may hit the skier at the head-trunk area due to the whiplash effect. Moreover, a reduction of wall thickness decreases pole damage speed, which 
increases the risk of pole fractures. For the current impact speeds in World Cup slalom races, advanced investigations are necessary for lowering the pole mass.

In children and youth races, skiing speed and thus impact speed is clearly lower than in World Cup races resulting in lower skier-pole impulse with lower maximum pole deflection and pole damage speed. Lower speeds permit reducing pole mass by lowering pole diameter and wall thickness. Assuming lower impact speeds of $2 \mathrm{~m} / \mathrm{s}$ than in World Cup races, pole d27-w2.5 showed the same maximum deflection as the World Cup approved pole d31-w3. Also the difference between impact speed and pole damage speed was the same for both poles. However, time loss would be reduced about $40 \%$ in the described seven gate flat course due to the mass reduction.

\section{Limitations}

In this study, variations of temperature or plasticity of the upright pole were not considered. A yielding movement of the skier at pole contact was not included. The dimension of the impactor was not varied. An impactor with a smaller diameter may reduce damage speed. Pole damage speed was not analyzed for the impact on the snow surface.

Acknowledgments The authors would like to thank the International Ski Federation for its financial support. The computational results presented have been achieved using the HPC infrastructure LEO of the University of Innsbruck.

\section{References}

1. The International Ski Federation (FIS) (2014) The international ski competition rules (ICR) Book IV Joint regulations for alpine skiing. http://www.fis-ski.com/mm/Document/documentlibrary/AlpineSkiing/03/29/54/ICR_clean_12082014_Neutral.pdf. Accessed 19 Sep 2016

2. The International Ski Federation (FIS) (2008) FIS specifications for flex poles. http://bmsi.ru/ media/75f004dd-d384-4d1f-aa99-0882bff649b3/kip08e.pdf. Accessed 12 Nov 2015

3. Innerhofer G, Nachbauer W (1999) Influence of the skier's mass and height on clearing slalom gates. Paper presented at the 10th European congress on sports medicine, University of Innsbruck, Innsbruck, 22-24 September 1999

4. Bere T, Flørenes TW, Krosshaug T, Haugen P, Svandal I, Nordsletten L, Bahr R (2013) A systematic video analysis of 69 injury cases in World Cup alpine skiing. Scand J Med Sci Sports 24:667-677

5. Schindelwig K, Ellensohn S, Kaps P, Nachbauer W (2016) Validation of a 3D finite element model for flex pole impacts. JSET (in review)

6. Gall K, Horstemeyer MF, Degner BW, McDowell DL, Fan J (2001) On the driving force for fatigue crack formation from inclusions and voids in a cast A356 aluminum alloy. Int J Fract 108:207

7. Brownlie L, Larose G, D'Auteuil A, Allinger T, Meinert F, Kristofic P, Dugase S, Boyde R, Stephens D (2010) Factors affecting the aerodynamic drag of alpine skiers. Procedia Eng 2:2375-2380 
8. The International Ski Federation (2016). http://www.fis-ski.com/alpine-skiing/events-andplaces/results/. Accessed 13 Apr 2016

9. Austrian Ski Federation (2016). https://www.oesv.at/media/media_vereinsservice/media_wettkampfordnungen/OeWO-ALPIN-2015-2016-fuer-Homepage.pdf. Accessed 13 Apr 2016

Open Access This chapter is distributed under the terms of the Creative Commons AttributionNoncommercial 2.5 License (http://creativecommons.org/licenses/by-nc/2.5/) which permits any noncommercial use, distribution, and reproduction in any medium, provided the original author(s) and source are credited.

The images or other third party material in this chapter are included in the work's Creative Commons license, unless indicated otherwise in the credit line; if such material is not included in the work's Creative Commons license and the respective action is not permitted by statutory regulation, users will need to obtain permission from the license holder to duplicate, adapt or reproduce the material. 